\title{
Meningeal sarcoidosis, pseudo-meningioma, and pachymeningitis of the convexity
}

\author{
D Ranoux, B Devaux, C Lamy, JY Mear, F X Roux, J L Mas
}

\begin{abstract}
Two cases of meningeal sarcoidosis with unusual and misleading presentations are reported. In the first case, CT scan, angiographic, and MRI findings were indistinguishable from those of meningioma. CSF pleiocytosis may help in diagnosing sarcoid pseudo-meningioma. The second patient had transient focal deficits and pachymeningitis of the convexity. The transient deficits were probably of epileptic origin based on their response to antiepileptic treatment. The diagnosis of neurosarcoidosis was made only after meningeal biopsy, despite thorough investigations.
\end{abstract}

Neurological symptoms, including cranial neuropathies, aseptic meningitis, hydrocephalus, intracranial masses, encephalopathies, seizures, peripheral neuropathies, and myopathies, develop in $2 \%$ to $5 \%$ of patients with sarcoidosis. ${ }^{1}$ Symptoms of meningeal involvement are protean and may take the form of subacute, chronic, or recurrent meningitis. Many patients are asymptomatic. ${ }^{12}$

We report two unusual and misleading presentations of sarcoid meningeal involvement. One mimicked a meningioma, both clinically and radiologically. The other patient had transient focal deficits and pachymeningitis of the convexity. The diagnosis was made only after meningeal biopsy despite thorough investigations.

\section{Case reports}

CASE 1

A 32 year old West Indian woman was admitted with a two month history of continuous left sided retro-orbital headache associated with paraesthesia of the left side of her face. Pulmonary and ocular sarcoidosis had been diagnosed in 1981, and she was being treated with prednisone $(100 \mathrm{mg} /$ day $)$ and dapsone (100 mg/day). Neurological examination revealed hypesthesia around the left fifth cranial nerve with decreased corneal reflex and a left ptosis. The erythrocyte sedimentation rate (ESR) was $23 \mathrm{~mm}$ in the first hour. The angiotensin-converting enzyme (ACE) activity and chest $x$ ray pictures were normal. A CT scan showed a slightly hyperdense homogeneous contrast-enhancing mass lying along side the left cavernous sinus (fig 1a). This lesion was hypointense and surrounded by oedema on axial T2-weighted MR images (fig 1b) and was enhanced on T1-weighted gadolinium MRI (fig 1c). Carotid angiography showed a slight stenosis of the C5 segment of the left internal carotid artery. Selective left external carotid angiography showed a vascular lesion lateral to the carotid siphon, supplied by the middle meningeal artery (fig 1d). Cerebrospinal fluid was normal except for elevated proteins $(0.85 \mathrm{~g} / \mathrm{l})$. A diagnosis of meningioma was made, and the patient was treated with carbamazepine.

She was readmitted 16 months later because of worsening headaches and diplopia. Examination showed trigeminal hypesthesia, ptosis and paresis of the medial rectus on the left. CT scans and MRI showed an increase in the size of the lesion. A craniotomy revealed a firm, whitish tissue which was adherent to the dura, extended to the cavernous sinus, and reached the sphenoid wing. The lesion, grossly resembling a meningioma, was partially removed. The histological examination showed multiple epithelioid granulomas without necrosis. Prednisone was increased to $50 \mathrm{mg}$ per day. The left ptosis, headaches, and diplopia resolved within a few days.

CASE 2

A 48 year old white woman had a four month history of recurrent episodes of left hemiparesis. These attacks of weakness were of acute onset, lasted five to 10 minutes, and were sometimes preceeded by a right temporal headache. They always affected the left arm and sometimes the left leg. In addition she experienced unrelated attacks of clonic movements of her left hand, lasting a few minutes. Her medical history was unremarkable. On admission examination revealed weakness and astereognosis of her left hand. An EEG showed paroxymal activity in the right temporal region, increased by hyperventilation. She received phenobarbitone and the attacks stopped. Plain CT scan (fig 2a) showed increased density of the gyri of the right temporo-parieto-occipital and left occipital regions, which enhanced after contrast. There was a large area of hypodensity in the white matter of the right hemisphere and left occipital region. T1-weighted MRI showed a slight hypointense signal of the white matter with mass effect. On T2-weighted sections the normal high signal intensity of the sulci was replaced by a hypointense signal, which enhanced after gadolinium injection (fig $2 b, c)$. CSF examination revealed a white cell count of $608 \mathrm{~mm}^{3}$ with $80 \%$ lymphocytes, $10 \%$ monocytes, and $8 \%$ neutrophils, a protein content of $1.30 \mathrm{~g} / \mathrm{l}$, and a glucose concentration of 
Figure 1a CT scan: contrast-enhancing mass (arrow) lying along left cavernous sinus. (b) T2-weighted MRI (2000/90); lesion (arrows) hypointense compared to nomal brain. (c)

T1-weighted MRI (SE 48/15) with injection of gadolinium; enhancement of lesion. (d) Selective external carotid angiography; lesion (arrow) fed by branches of middle meningeal artery.
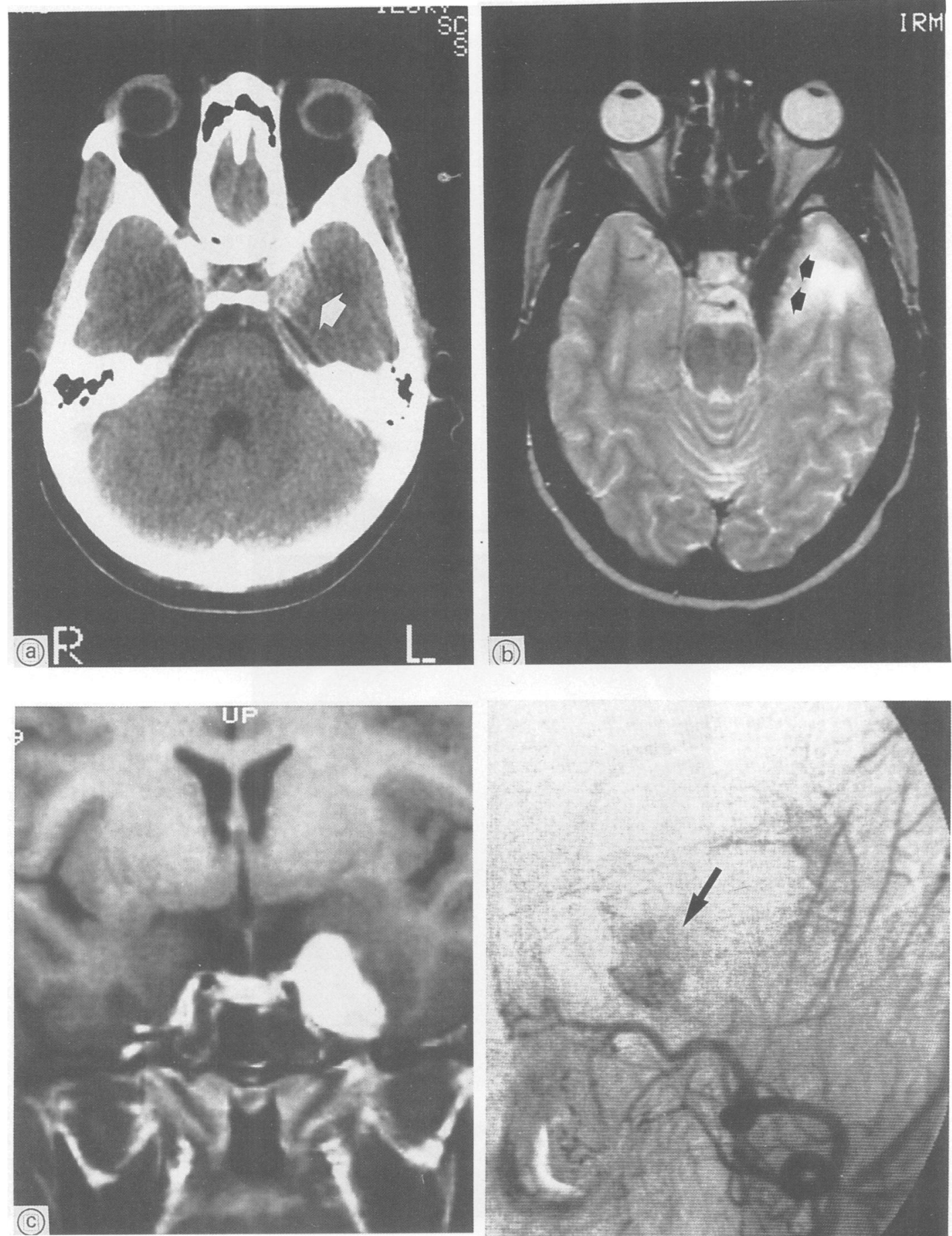

$1.84 \mathrm{mmol} / \mathrm{l}$. CSF cultures for acid-fast organisms and fungi, india ink preparation, and cryptococcal antigen study were negative. The ESR was $23 \mathrm{~mm}$ in the first hour. ACE concentration in serum and CSF, tuberculin test (10 units), liver and salivary gland biopsy specimens, thoracic CT scan, fibreoptic bronchoscopy, Gallium scintigraphy, and extensive serological studies all yielded normal or negative results. The pulmonary function tests showed a significant decrease in the diffusion capacity. Despite the negativity of the acid-fast organisms cultures, the patient was treated with antituberculous drugs.

A meningeal and cortical biopsy was performed two months after admission. The dura was tense and adherent to the cortex. The brain surface was yellowish and rubbery.

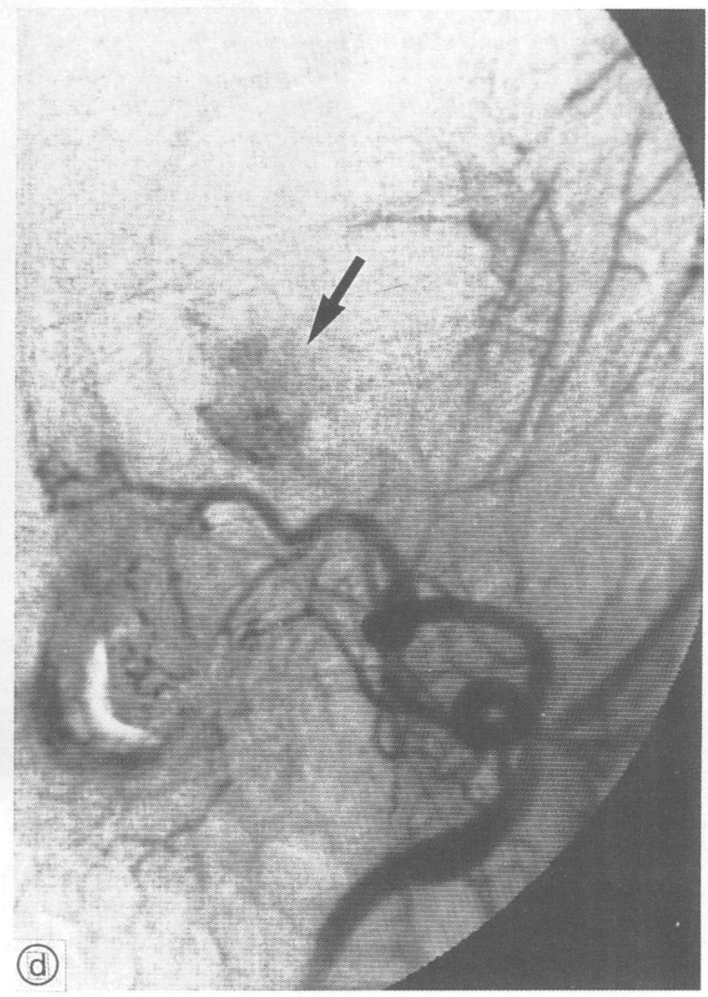

Microscopic examination revealed a fibrous thickening of the meninges and nodular granulomatous lesions, without central necrosis. Some follicles had a perivascular distribution. The walls of some vessels were invaded by lymphocytes with disruption of the internal elastic lamina. Cultures for bacteria, acid-fast organisms, and fungi were negative. The cortical samples showed no abnormality. These findings were consistent with the diagnosis of sarcoidosis. The patient was treated with prednisone ( $1 \mathrm{mg} / \mathrm{kg}$ per day) with continuation of 

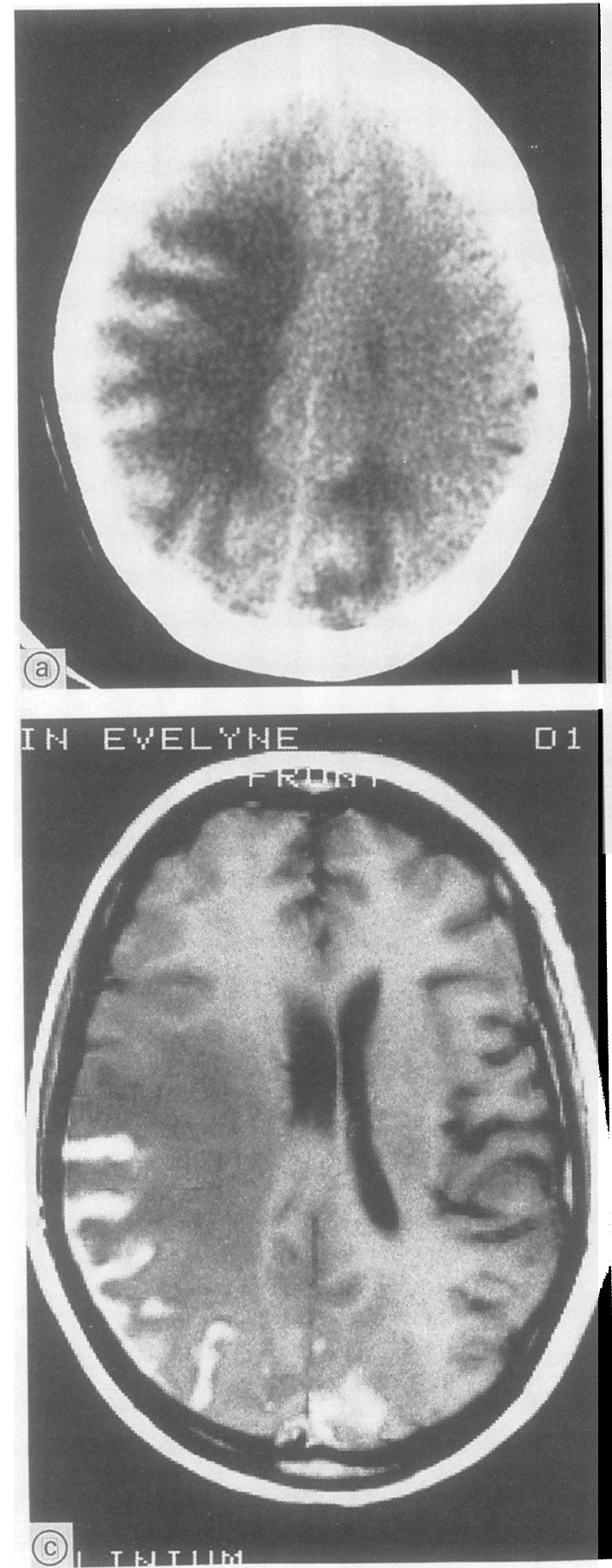

antituberculous therapy. Two months later her clinical examination was normal. A CT scan and MRI showed a clear decrease in the size of white matter lesions and the persistance of the low signal in the affected sulci.

\section{Discussion}

The diagnosis of meningeal sarcoidosis in case 1 was preoperativally mistaken for meningioma. This had also occurred in the few previously reported cases of sarcoid pseudomeningioma. $^{2-8}$ Radiological investigations cannot differentiate between the two diseases. In all cases of sarcoid pseudo-meningioma, CT scan showed an homogeneously enhancing mass and, in two cases, ${ }^{78}$ skull $x$ ray pictures revealed hyperostosis of the sphenoid wing. In

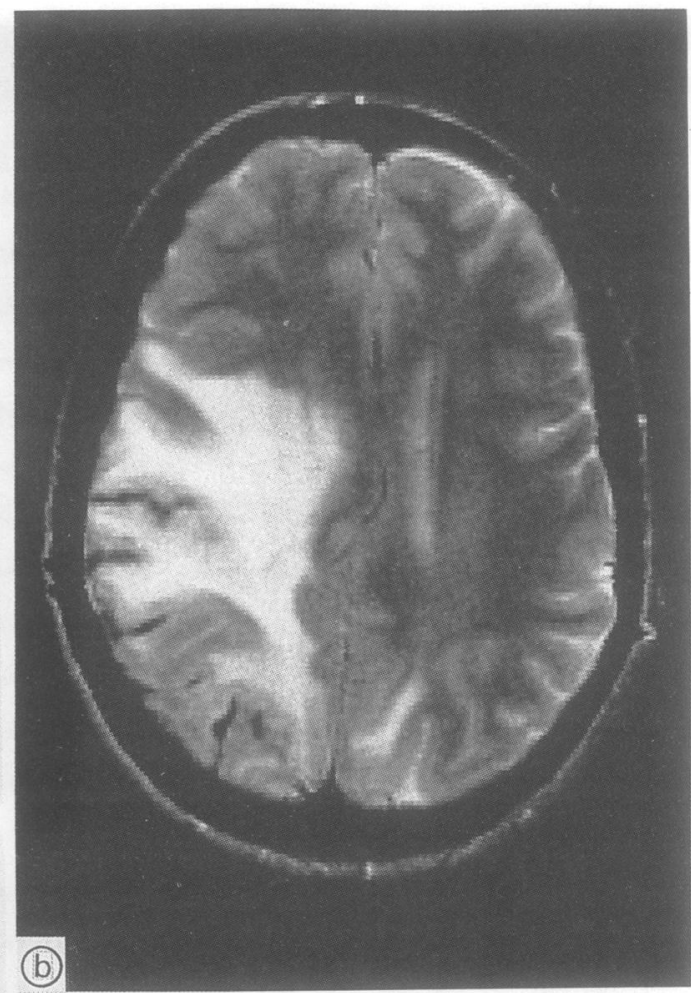

Figure $2 a \quad C T$ scan without contrast; spontaneous increased density and thickening of sulci of right temporo-parieto-occipital and left occipital regions. Large hypodensity in white matter. (b) T2-weighted MRI (2200/90); large area of hypersignal in white matter with mass effect. Normal signal of sulci replaced by hypointense signal. (c) T1-weighted images MRI (600/15) with injection of gadolinium; enhancement of sulci corresponding to hypointense linear images seen on $T 2$ sequences.

addition, selective external carotid angiography may reveal a blush, ${ }^{8}$ as seen in our first case. MRI findings in sarcoid pseudo-meningioma have not been reported. In case 1 , the lesion was clearly hypointense on T2-weighted sequences and enhanced after admininstration of gadolinium. Meningiomas are usually hyperintense or isointense of T2-weighted sequences but $9 \%$ to $18 \%$ are hypointense. CSF examination may help in diagnosing sarcoid pseudo-meningioma when it reveals a pleocytosis, ${ }^{24}$ which has not been reported in meningiomas. ${ }^{10}$ In case 1 , the CSF cell count was normal, but the patient was receiving corticosteroids at the time of the lumbar puncture. We suggest that patients with a known sarcoidosis and evidence of an antracranial mass undergo a lumbar puncture (if not contraindicated by the volume of the lesion) and receive a trial of steroids before surgery. Radical removal may be justified only if the lesion is causing important neurological impairment.

Pachymeningitis of the convexity is a rare presentation of neurosarcoidosis. ${ }^{11-16}$ Headaches are the most common clinical feature followed by seizures. Two patients have presented with transient focal deficits ${ }^{16}$ (case 2). In view of the prevalence of affected small vessels and subclinical infarctions at necropsy 
of patients with neurosarcoidosis ${ }^{17}$ an ischaemic mechanism must be considered. In our second case, however, an epileptic origin is more likely because the attacks of transient focal deficit disappeared with anticonvulsants, and the patient had also unrelated episodes of focal seizures. Negative phenomena of epileptic origin are rare but can occur especially in parietal lobe lesions. ${ }^{18} \mathrm{CSF}$ has shown nonspecific elevation of the protein content or pleocytosis, or both. On CT scan granulomatous thickened meninges appear as slightly increased density with moderate or dense homogeneous enhancement. This aspect has been reported in pachymeningitis with various causes. ${ }^{19}$ The clear gyral enhancement seen in case 2 is probably explained by extension of the meningeal infiltrative process through the Virchow-Robin spaces. ${ }^{13}$ In two patients with sarcoid pachymeningitis of the convexity ${ }^{20}$ MRI has shown an isointense signal relative to brain on T1-weighted sequences and heterogeneously hyperintense or predominantly hypointense on T2-weighted sequences. The very hypointense signal of the sulci observed in case 2 on T2-weighted sequences may be related to the fibrous thickening of the meninges noted at the microscopic examination of the meningeal biopsy specimen.

The diagnosis of sarcoidosis is difficult when neurological involvement is the first or only manifestation of the disease. In fact no reliable diagnostic test for neurosarcoidosis exists. The yield from liver, salivary gland, transbronchial or muscular biopsy specimens in the diagnosis of clinically isolated neurosarcoidosis has not been evaluated. These specimens may remain negative in histologically-proven neurosarcoidosis. Even necropsy may fail to reveal systemic disease. ${ }^{21}$ Our second case, as well as other cases reported emphasise the difficulty of substantiating the diagnosis of neurosarcoidosis without cerebral or meningeal biopsy. Making the diagnosis early in the course of the disease is important as the development of meningeal fibrosis may limit response to steroids.

1 Stern BJ, Krumholz A, Johns C, Scott P, Nissim J. Sarcoidosis and the neurological manifestations. Arch Neurol 1985;42:909-17.

2 Cahill DW, Saloman M. Neurosarcoidosis: a review of the rarer manifestations. Surg Neurol 1981;15:204-11.

3 Brooks BS, El Gammal T, Hungerford GD, Acker J, Trevor $\mathrm{RP}$, Russel W. Radiological evaluation of neurosarcoidosis: role of computed tomography. AfNR 1982, 3:513-21.

4 Goodman SS, Margulies ME. Boeck's sarcoid simulating a brain tumor. Arch Neurol Psych 1959;81:419-23.

5 Osenbach RK, Blumenkopt B, Ramirez H, Gutierrez J. Meningeal neurosarcoidosis mimicking convexity enplaque meningioma. Surg Neurol 1986;26:387-90.

6 Skillicorn SA, Garrity RW. Intracranial Boeck's sarcoid tumor resembling meningioma. $\mathcal{f}$ Neurosurg 1955; tumor rese

7 Gudeman SK, Selhorst JB, Susac JO, Waybright EA Sarcoid optic neuropathy. Neurology 1982;32:597-603.

8 Frisen L, Lindgreen BJ, MacGregor JL, Stattin S. Sarcoidlike disorder of the intracranial optic nerve. $\mathcal{F}$ Neurol Neurosurg Psychiatry 1977;40:702-7.

9 Elster AD, Challa VR, Gilbert TH, Richardson DN, Contento JC. Meningiomas: MR and histopathologic features. Radiology 1989;170:857-62.

10 Adams RD, Victor M. Principles of neurology. 2nd ed. New York. McGraw-Hill, 1981 .

11 De Tribolet N, Zander E, Phil D. Intracranial sarcoidosis presenting angiographically as a sub-dural hematoma. presenting angiol 1979;9:169-71.

12 Healton EB, Zito G, Chauhan P, Brust JC. Intracranial subdural sarcoid granuloma. $f$ Neurosurg 1982; 56:728-31.

13 Mirfakhraee M, Crafford MJ, Guinto FC, Nauta $H$, Werdn VW. Virchow-Robin space: a path of spread in neuroVW. Virchow-Robin space: a path of sp
sarcoidosis. Radiology 1986;158:715-20.

14 Pépin B, Bacri D, Haguenau $M$, Chai $N$, Lougnon J, Woimant $F$. Sarcoidose à manifestations cérébrales révélaWoimant F. Sarcoidose à manifestations
trices. Ann Med Interne 1979;130:185-9.

15 Powers JM. Sarcoidosis of the tentorium with cortical blindness. F Clin Neuro-Ophtalmol 1985;5:112-5.

16 Sethi KD, El Gammal T, Patel BR, Swift TR. Dural sarcoidosis presenting with transient neurologic symptoms. Arch Neurol 1986;43:595-7.

17 Herring AB, Urich $\mathrm{H}$. Sarcoidosis of the central nervous system. F Neurol Sci 1969;9:405-22.

18 Lee H, Lerner A. Transient inhibitory seizures mimicking crescendo TIAs. Neurology 1990;40:165-6.

19 Moore AP, Rolfe EB, Jones EL. Pachymeningitis cranialis hypertrophica. I Neurol Neurosurg Psychiatry 1985; 48:942-4.

20 Hayes WS, Sherman JL, Stern BJ, Citrin CM, Pulaski PD.

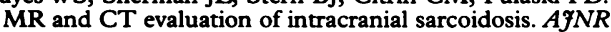
MR and CT eval
1987;8:841-7.

21 Aszkanazy CL. Sarcoidosis of the central nervous system. $\mathcal{f}$ Neuropathol Exp Neurol 1952;11:392-400. 